\title{
A Novel Way of BPA Calculation for Context Inference using Sensor Signals
}

\author{
Shinsook Yoon $^{3}$, Chang-Keun Ryu ${ }^{2}$ and Donghyok Suh ${ }^{*}$ \\ ${ }^{3}$ Department of Information and Communication, Korea Nazarene University \\ ${ }^{2}$ Department of Electronics, Namseoul University \\ ${ }^{1}$ Department of Multimedia, Far East University, South Korea \\ 3yss28@daum.net\}, ${ }^{2}\{c k r y u @ n s u . a c . k r\},{ }^{1}\{$ hanhwaco@kdu.ac.kr\}
}

\begin{abstract}
Basic probability assignment (BPA) has the key role in multi-sensor data fusion. This paper proposed a way to determine BPA using the various signals acquired from the sensors. It described the analysis of signals detected by the sensors. The determined $\hat{B} P A$ were used for multi-sensor data fusion to infer and recognize the context targeted by a wireless sensor networks. To determine BPA, the change rate was calcylated and assessed to be reflected. The method enabled context inference using the sensor signals even when there was no
\end{abstract} advanced information of the situation.

Keywords: Context inference, Basic Probability Assgnment, Dempster-Shafer Theory, multi-sensor data fusion

\section{Introduction}

There have been many studies of recognizing the sensor attached objects and surrounding environments by forming a wireless sensor networks and acquiring the data detected by those sensors. Heterogeneous sensors detect the changes of situations using different functions. Compared to the network system consisting of single sensor, the network system of heterogeneous sensors can deliver better context data. To infer the situation, the signals acquired from the sensors are processed through the multi-sensor data fusion. Methods used in multi-sensor data fusion rncrude the statistical analysis, neural network, Kalman filter, and fuzzy theory. The Kalman filter is widely used for inferring the location of moving objects while the neural network method is based on the learning theory. Dempster-Shafer Theory (DST) of the statistical analysis and fuzzy theory require BPA. Ontology has been widely used in existing studies of context awareness using wireless sensor network. It is a method of acquiring the context data and modeling it in advance and then matching the data from the multi-sensors to the model. The weakness of this method is that the system would not be able to recognize anything beyond what it modeled in advance. As the real world is so diverse, it would not be possible to perfectly model all possible situations in advance. Data fusion with DSTMs beneficial for context inference without any advanced information. Data fusion using DST can calculate the belief and plausibility of each focal element using BPA. DST is dependent on the BPA. How can BPA be calculated for context awareness when there are no data and thus no model in advance? We have to calculate BPA to infer the situation. Then we should consider the factors how the signals from the sensors relate to construct the context.

*Corresponding Author 
BPA, which is assigned to the elements structured of sensing data, must reflect the assessed level of contribution to the context by the signals. We propose a method of sensing signal assessment suitable for context inference and present the way to infer the situation by reflecting the sensing signal assessment. The objective of this study is to enable context inference even for the case of exceeding the scope of advance model using it.

This paper is organized as follows: In Chapter 2, the related studies are described. In Chapter 3, a way to determine BPA is proposed. In Chapter 4, the experiment and its evaluation are described. Lastly, Chapter 5 presents conclusion.

\section{Related Research}

Studies of multi-sensor data fusion have recently gained much attention in many fields. DST and fuzzy theory are important methods of data fusion. They have also been used in many studies, how to determine BPA, which are the important elements of DST and fuzzy theory.

\subsection{Dempster-Shafer Theory and Basic Probability Assignment}

DST is useful for situation reasoning through multi-sensor data fusion. DST is a probability theory articulating the uncertainty of real world Heterogeneous elements can be blended using DST. It contains the focal elements and BPA is assigned to each of them. Then the belief and plausibility of each focal element are calculated using the assigned BPA. BPA has the following properties $[6,7]$ :

$$
m: 2^{\theta} \rightarrow[0,1], m(\varnothing)=0,0 \leq m(A) \leq 1 \operatorname{and} \underset{A \leq \theta}{D} m(A)=-
$$

Provided that is defined as a univers set, which consists of the exclusive propositions and is the number of power set, ncorporating all the possible combination of the propositions. The $m$ Is the Basic Probability Assignment. The value bel(A), is a belief function equivalent to the assignment values of the quantified total belief for combination, as shown below.

$$
\text { bel }(A)=\sum_{x=A} \cap(A) \text {, for all } A \equiv \theta
$$

The BPA is calculated based on the values of the total fusion system. Fusing the BPA values, $m_{1}, m_{2}, \ldots, m_{n}$ Independently calculated from $\mathrm{n}$ modules, yields the following.

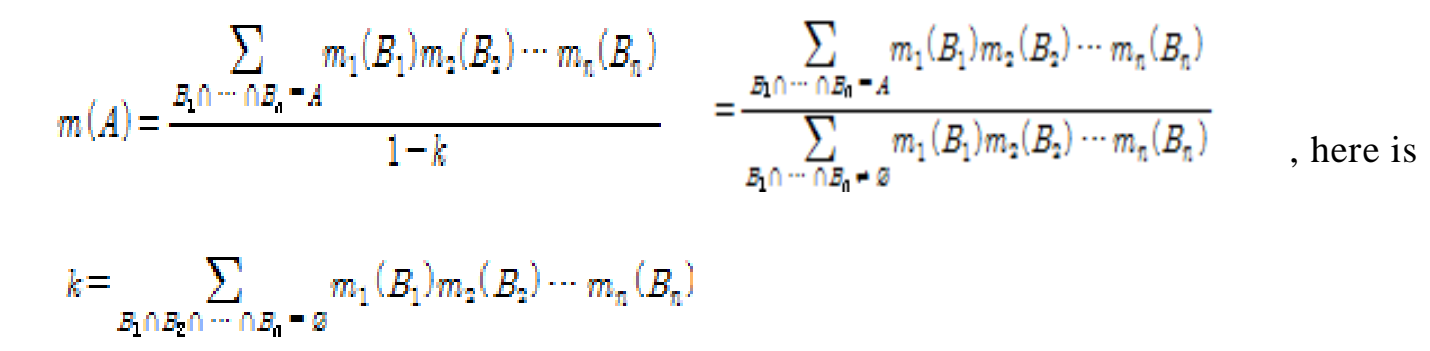

Using the calculated belief and plausibility, it helps to assess each element of interest. 


\subsection{Previous Works}

T. Ali and P. Dutta proposed a method to determine BPA when minimum, maximum and most likely values of the parameter are known. They developed an extended version of uncertainty measurement in evidence theory in order to calculated total uncertainty of the body of evidence [1]. A.O. Boudraa, A. Bentabet, F. Salzenstein and L. Guillon proposed an image segmentation method based on DST. BPA is estimated in unsupervised way using pixels fuzzy membership degrees derived from image histogram [2]. Z. Zuo, Y. Xu and G. Chen proposed a method of rough set theory based on random set and BP neural network to obtain the BPA [3]. Wen Jiang proposed a new method to determine BPA based on the distance measure between the sample data under test and the model of attributes of species [4].

\section{Determination of BPA in Multi-sensors Data Fusion}

The wireless network of heterogeneous sensors is better than the network of single type sensors. That is because the real situations to be recognized are diverse and the elements of the situations are also in different forms. Sensors with different functions detect and report the situations differently, and that is why the multi-sensor data fusion is needed.

Multi-sensor data fusion is targeted to heterogeneous data. To process the heterogeneous data, they must first be converted to the same baseline. Fourier transform can be used for that. It transforms a real world signal into a polynomal expression having the sine or cosine function as the basic function. Transforming the signals of different nature into the expressions having the same basic function will enable composition and manipulation of the signals. BPA described in this paper is significant in that it transforms the heterogeneous signal values or data into the values of same nature to be blended.

\subsection{Determination of BPA for Context Inference}

For assess the signals from the sensors and get the BPA, this study assumes the purpose of determining BPA is to infer the context and that there are no data available in advance. The procedure of determine BPA of eacn focal element is as follows:

First, the ways to assess the signals detected and reported by the sensors are decided.

Second, the ways to reflect the signals from the sensors are defined when composing the elements of interest.

Third, signals reported by the sensors are assessed and normalized to conform to the basic properties of BPA.

This study focuses on how to assess the signals reported by the sensors.

\subsubsection{Sensor and Signal}

Each nerminal node of wireless sensor networks has the built-in sensor. A sensor mote consists of the sensor unit to detect the surrounding environment, data processing unit, power supply unit, and communication unit to communicate with the network. The sensor detects the change of the surrounding environment and decides whether it should be reported to the host or not. The sensed data are reported to the host through the relay nodes and sync nodes. The sensors sense at certain intervals which are determined by the user. The signals detected and reported by the sensors are analog signals. The analog signals are converted into the digital values by the A/D converter before they are transferred to the host. Sensing signals transferred in digital form are assessed with consideration to context. 


\subsubsection{Assessment of Signal}

Signals sensed and reported by the sensors should be assessed in terms of change with time. A context is more likely to be variable than fixed, and the change of the context mostly occurs over time. When there is no information in advance, sudden change of the sensing value must be noted. Assessment of the change of sensing value may have different guideline according to the type of the sensor.

Sensors are not measured continuously. Even when the values are continuously measured, the continuous analog signal is converted into the digital signal by the A/D converter before it is reported. From the host's point of view, the values reported by the sensor are the discrete data. As more data are reported, the accumulated sensor measurements may show a specific pattern. We assess the acquired signals using such guideline and reflect such assessment in BPA calculation.

Assessment of Variation Rate
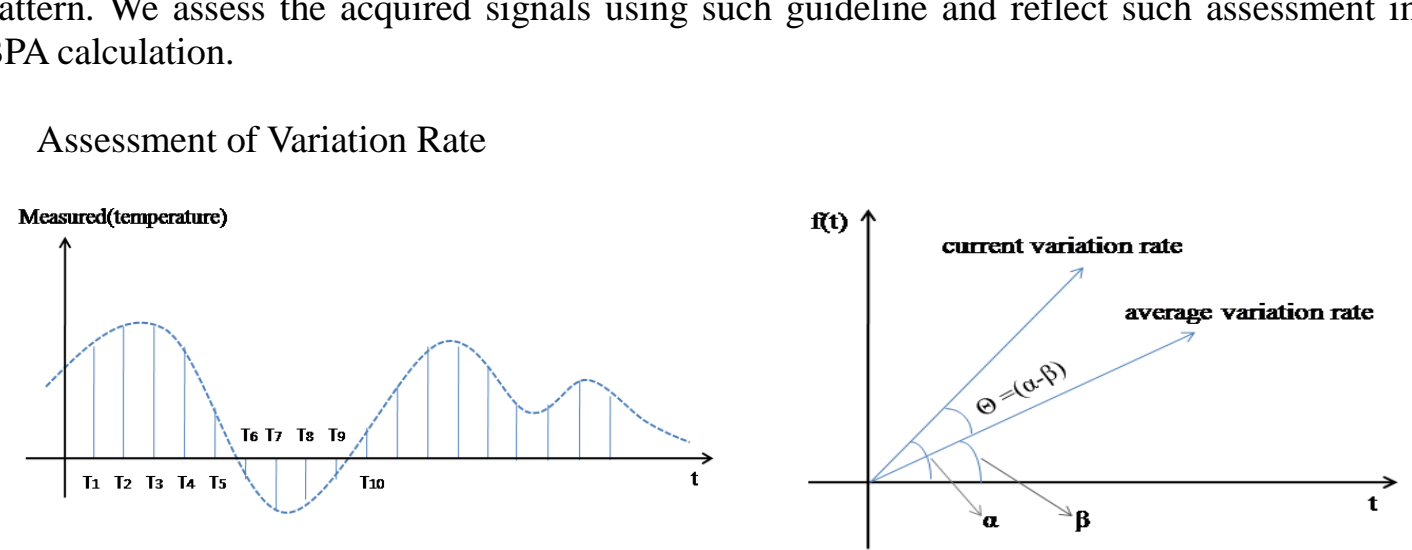

Figure 1. (a) Reported Values

The sensed temperature is $f(t)$, and $F_{n}=f\left(t_{n}\right) f\left(t_{0}\right)$, the average variation rate is $\frac{1}{n} \sum_{k=1}^{n} F_{k}$ for the time $t_{0}, t_{1}, t_{2}, \ldots t_{n}$.

As the variation rate is the linear function that passes $(0,0)$ and $(\operatorname{tn}, f(\operatorname{tn}))$, we can measure the difference between the current rate and average rate with the angle $\theta=\alpha-\beta$. So, we can make use of this angle for assessment of the sensed values.

$$
\int \tan \theta_{n}=\left|\frac{\tan \alpha-\tan \beta}{1+\tan \alpha \tan \beta}\right|=\left|\frac{F_{n}-\frac{\sum_{k=1}^{n} F_{n}}{n}}{1+F_{n} \cdot \frac{\sum_{k=1}^{n} F_{n}}{n}}\right|
$$

For $\tan \theta$ have value in $[0, \infty]$, it can transformed $\sin \theta_{n}$ that have value in the range $[0,1]$

$$
\tan \theta_{\mathrm{n}}=\frac{\sin \theta_{\mathrm{n}}}{\sqrt{1-\sin ^{2} \theta_{\mathrm{n}}}}, \text { so, } \sin \theta_{\mathrm{n}}=\sqrt{1-\frac{1}{1+\tan ^{2} \theta_{\mathrm{n}}}}
$$

Set $\sin \theta_{n}$ is $\alpha_{n}$, we can define the $A_{n}$ with the $f(t)$ sent by temperature sensor. We can derive $A_{n}$ for mass function,

$$
\mathrm{A}_{\mathrm{n}}=\frac{\alpha_{\mathrm{n}}}{\sum_{\mathrm{k}=1}^{\mathrm{n}} \alpha_{\mathrm{k}}}
$$


We can calculate $B_{n}, C_{n}$, as the mass function of the other sensors with the same way. We showed the way of assess the signals to determine BPA. BPA should assign to each focal element for the multi-sensors data fusion with DST. In DST, the focal element can be considered as 'probable events', 'factors affecting the context' or 'assumption'. They are the elements of the context to be inferred with wireless sensor networks. The multi-sensors data fusion processing can be enabled even with the heterogeneous elements if BPA is assigned to each focal element.

\section{An Experiment and Evaluation}

We adopted temperature, illumination and sonic sensors. The host received the value from sensors every 10 seconds. Table 1 shows various values from 3 kinds of sensors at each 10 seconds. The 3 kinds of sensors are temperature, illumination and sonic. They sensed meaningful variations and reported them as events to the host through the sink node.

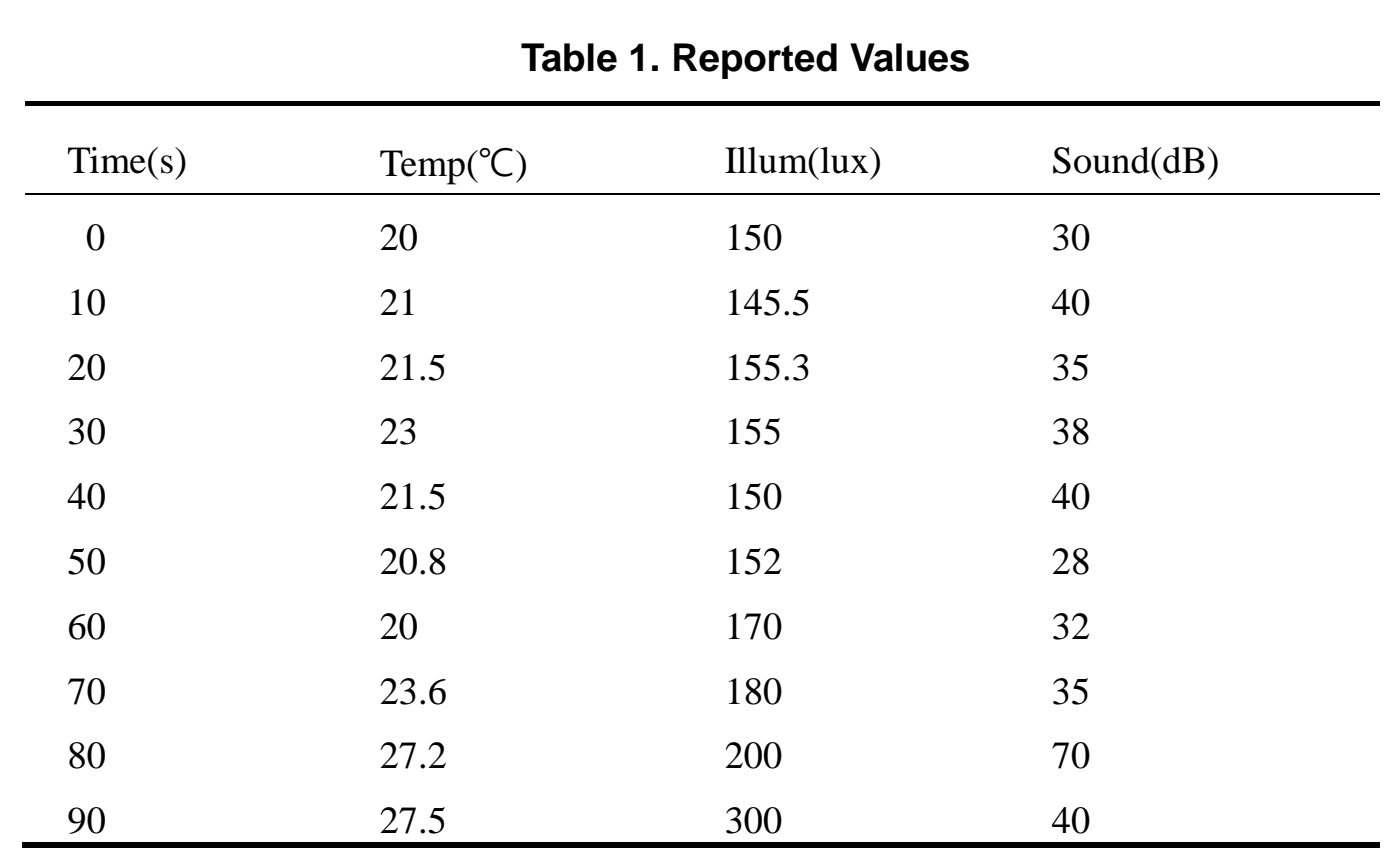

We can calculate the variation rate with the reported values. Table 2 shows the variation rate at its timeslot.

Table 2. The Variation Rate at its Timeslot

\begin{tabular}{llll}
\hline Time(s) & $\operatorname{Temp}\left({ }^{\circ} \mathrm{C}\right)$ & Illum(lux $)$ & Sound(dB) \\
\hline 0 & 0 & 0 & 0 \\
10 & 0.073 & 0.120 & 0.174 \\
20 & 0.079 & 0.133 & 0 \\
30 & 0.132 & 0.113 & 0.086 \\
40 & 0.009 & 0.065 & 0.111 \\
50 & 0.049 & 0.040 & 0.206 \\
\hline
\end{tabular}




\begin{tabular}{llll}
\hline 60 & 0.109 & 0.131 & 0.116 \\
70 & 0.156 & 0.129 & 0.011 \\
80 & 0.200 & 0.130 & 0.260 \\
90 & 0.188 & 0.134 & 0.033 \\
\hline
\end{tabular}

We made the focal elements with the combination of three sensors. $\varphi, h_{1}, h_{2}, h_{3}, h_{1} \cup h_{2}$, $\mathrm{h}_{1} \cup \mathrm{h}_{3}, \mathrm{~h}_{2} \cup \mathrm{h}_{3}, \Omega$ and are focal elements in this case. $\left(\mathrm{h}_{1}\right.$ : Temperature sensor, $\mathrm{h}_{2}$ : Illuminated sensor, $\mathrm{h}_{3}$ : Sound sensor) We can derive the fusion object with the variation rate. Table 3 shows the result of the belief and plausibility from the BPA of each focal element [5]. Table 4 shows the final result of the data fusion processing.

Table 3. The BPA, belief and plausibility of Two Fusion Object

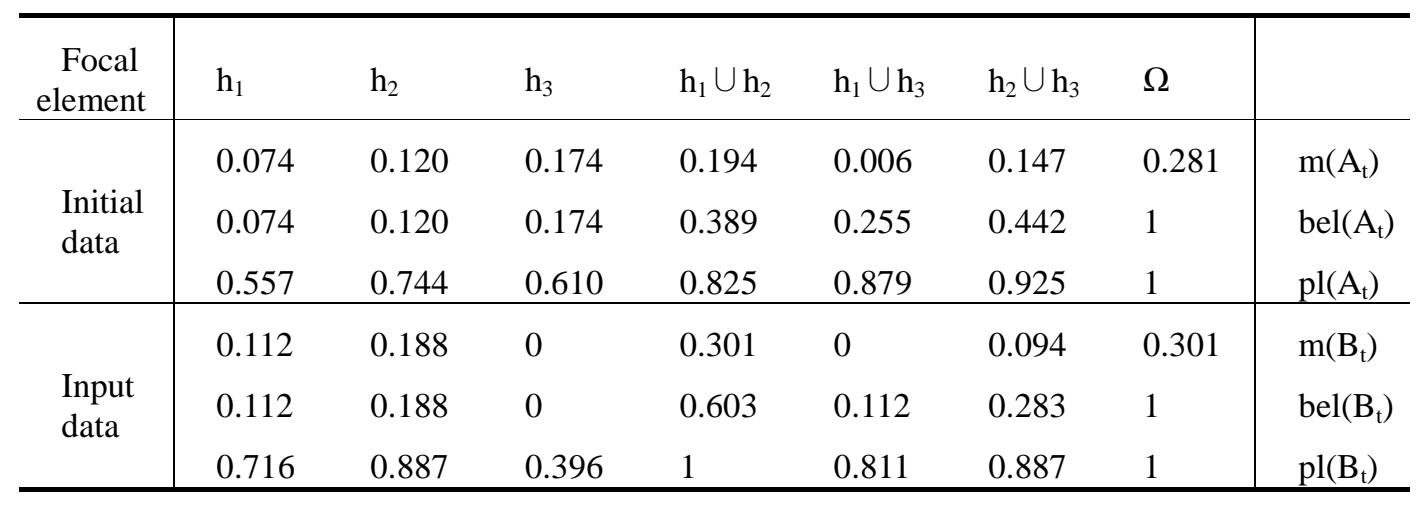

Table 4. The Calculated Belief and Plausibility

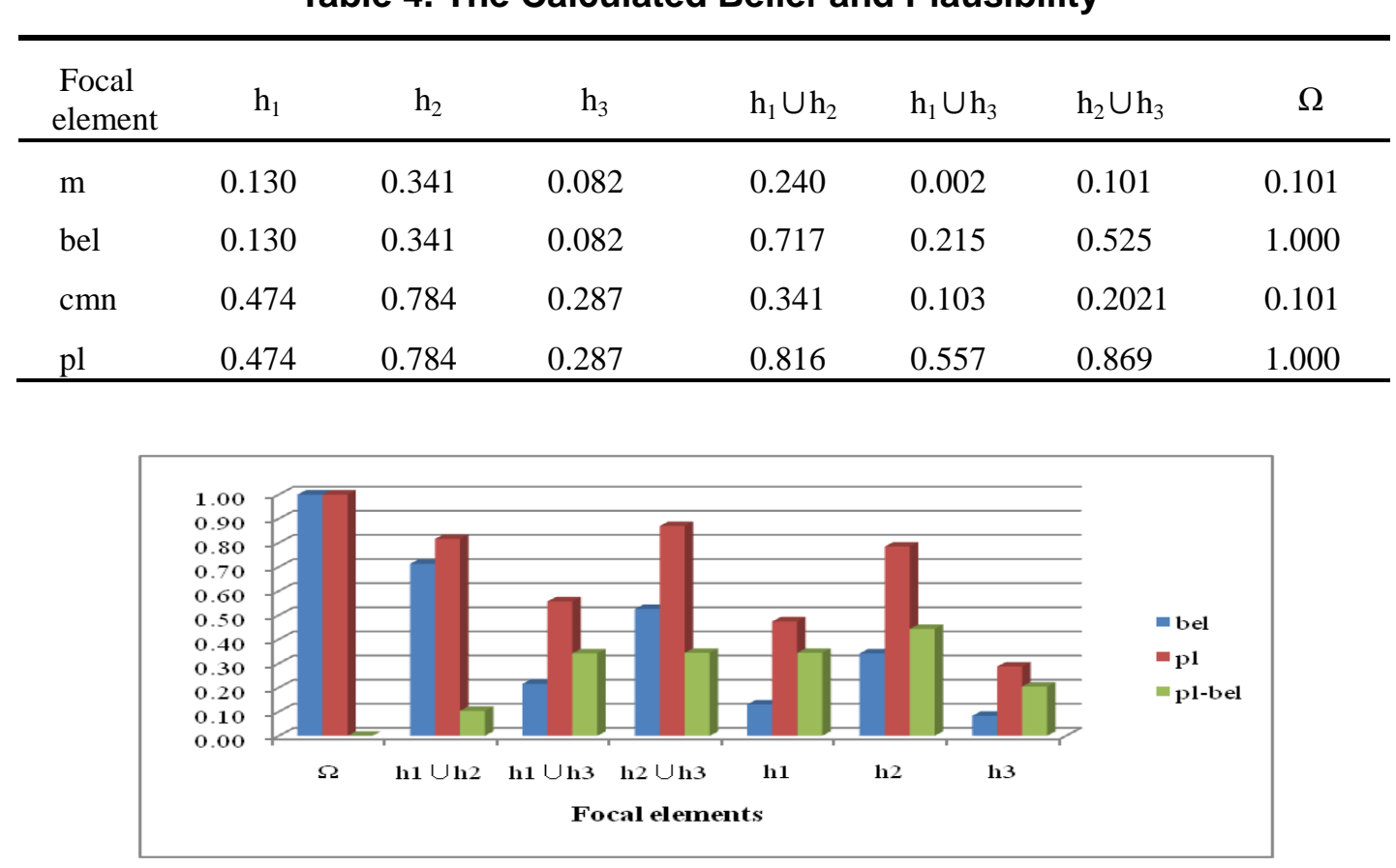

Figure 2. The Fusion Result 
This chapter describes the experiment of assessing the signals acquired through the sensors and reflecting the assessment in BPA. The experiment is conducted in the following procedure:

1. A wireless sensor network is constructed using 3 types of sensors.

They are temperature, illumination and sonic.

2. Signals reported to the host by each sensor are assessed in the way proposed in Chapter 3.

3. BPA of each element of interest is calculated based on the assessment.

4. DST based multi-sensor data fusion is calculated using BPA.

5. The context is inferred referring to the belief and uncertainty of each element of interest.

\section{Conclusion}

The wireless sensor network is constructed using the multi-sensors obtain clearer information. The context can be inferred using the neasured values sentby the sensors, and the multi-sensor data fusion is used for that. In this paper, we proposed a way to determine BPA using the signal values detected by the sensors to infer the context. Context reasoning without having the information in advance is needed becatse $i$ is impossible to obtain the information of various situations in real world in advance. Since the signals detected by the sensors affect the context or related to elements of the Context, the signals must be assessed with consideration to the changes and nature of the sensing values when determining BPA.

We assessed the signals detected and reported by the sensors by checking the change rates at specific time intervals and presented a way to analyze the pattern of the sensor measured values to assess them based on the dispersion with reference to a specific value. Since the signals detected by the sensots are related to the elements of the context, the experiment proved that the assessment of sensing values helped context reasoning. BPA determination through signal assessment proposed in this paper is significant that it can be used for context reasoning and context awareness even when there is no information in advance.

\section{Acknowledgements}

Funding for this paper was provided by Namseoul University.

\section{References}

[1] T. Ali, and P. Dutta, "Methods to Obtain Basic Probability Assignment in Evidence Theory", International Journal of Computer Applications, vol. 38, no. 4, (2012), pp. 46-51.

[2] A. O. Boudraa, A. Bentabet, F. Salzenstein and L. Guillon, “ Dempster-Shafer's Basic Probability Assignment Based on Fuzzy Membership Functions", Electronic Letters on Computer Vision and Image Analysis, vol. 4, no. 1, (2004).

[3] Z. Zuo, Y. Xu and G. Chen, "A New Method of Obtaining BPA and Application to the Bearing Fault Diagnosis of Wind Turbine", Proceedings of the 2009 International Symposium on Information Processing (ISIP'09), (2009) August, pp. 368-371.

[4] W. Jiang, "A New Method to Determine BPA in Evidence Theory", Journal of Computers, vol. 6, no. 6, (2011) June.

[5] U. Rakowsky, "Fundamentals of Dempster-Shafer theory and its applications to system safety and reliability modeling”, RTA, (2007), pp. 3-4.

[6] A. P. Dempster, "New Methods for Reasoning towards Posterior Distributions based on Sample Data", The Annals of Mathematical Statistics, vol. 37, (1996), pp. 355-374. 
[7] A. P. Dempster, "Upper and Lower Probablities Induced by a Multivalued Mapping", The Annals of Mathematical Statistics, vol. 38, (1967), pp. 325-339.

[8] V. N. Hamed, Z. Kamran and N. Nasser, "Context-Aware Middleware Achitecture for Smart Home Environment”, IJSH, 7, (2013) January 1, pp. 77-86.

[9] W. Mo and Z. Ding, "A Novel Template Weighted Match Degree Algorithm for Optical Character Recognition”, IJSH, vol. 7, no. 3, (2013) May, pp. 261-270.

[10] H. Kim, "Diversity of Mobile Distribution Systems", IJSH, vol. 7, no. 3, (2013) May, pp. 355-364.

\section{Authors}

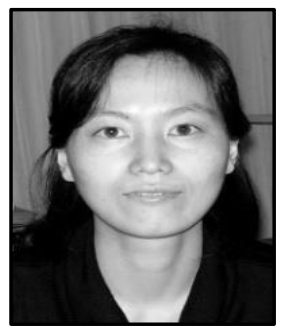

Shinsook Yoon, she received the M.S. degrees in computer engineering from Hoseo University in 2008. Her research interests included in stream data processing and data fusion in wireless sensor network.

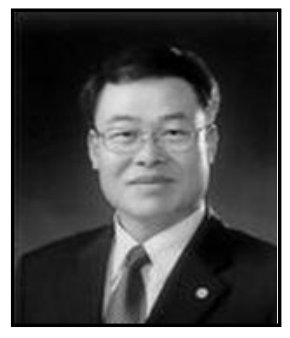

Chang-Keun Ryu, he is a professor at Namseoul University. He received the B.S. degree in electronicengineering from Dankook University in 1981, and the M.S. and Ph.D. in electronic engineering from Dankook University in 1993. His research interests are included in data fusion in wirelessensor network and system architecture.

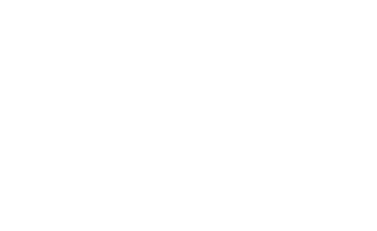

Donghyok Suh, he received the M.S. degrees in computer

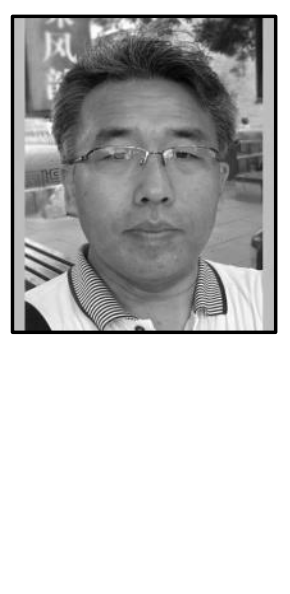
engineering from Hoseo University in 2005 and the Ph.D. in computerscience from Chungbuk National University in 2012. His research interests included in stream data processing and data fusion in wireless sensor network. 